\title{
Chronic suppurative mandibular osteomyelitis presenting as soft tissue swelling and trismus: A case report
}

\author{
Ahmad K, ${ }^{1 *}$ Ansari S, ${ }^{1}$ Dhungel $K,{ }^{1}$ Gupta MK, ${ }^{1}$ Amanullah $M F,{ }^{1}$ Kumar $A,{ }^{1}$ Rauniyar $R K^{1}$ \\ ${ }^{1}$ Department of Radiodiagnosis and Imaging, B.P. Koirala Institute of Health Sciences, Dharan, Nepal
}

\author{
*Corresponding Author: \\ Dr. Kaleem Ahmad, \\ Associate Professor, \\ Department of Radiodiagnosis, \\ B.P. Koirala Institute of Health Sciences, \\ Dharan, Nepal. \\ E mail address: drkalim17@yahoo.co.in \\ Citation \\ Ahmad K, Ansari S, Dhungel K, Gupta MK, Amanullah \\ MF, Kumar A, Rauniyar RK. Chronic suppurative \\ mandibular osteomyelitis presenting as soft tissue \\ swelling and trismus: A case report. Nepal Journal of \\ Medical Sciences 2013;2(2):197-9.
}

\begin{abstract}
Osteomyelitis of the mandible is a rare condition and it could be a serious complication of untreated odontogenic infection. Classically, patient with osteomyelitis of the mandible would experience pain and swelling over the affected side of the face. CT is usually indicated when there is extension of the infection into the adjacent soft tissue and fascial spaces which could be the presenting clinical symptom. Hereby, we present a case of mandibular osteomyelitis in an 11 year old girl who presented with pain and swelling in left lower jaw followed by extraction of tooth, diagnosed on CT followed by surgical management.
\end{abstract}

Keywords: Mandible; osteomyelitis; suppurative

\section{Introduction:}

Osteomyelitis is an acute or chronic inflammatory process that can involve cortical and trabecular aspects of bone or bone marrow. ${ }^{1}$ Moreover, osteomyelitis of the head and neck skeleton is rare, particularly in the jaws. ${ }^{2,3}$ This article reports a case of a healthy patient who developed osteomyelitis of the lower jaw following extraction of tooth. Local conditions that adversely affect the blood supply or lead to tissue necrosis can also predispose the host to a bone infection or localized osteomyelitis., ${ }^{4,5}$

\section{Case report:}

An 11 year old girl presented with long standing pain and swelling in the lower tooth region for the last 6 months for which she was treated by many dental practitioners, but there was no relief. Pain was dull, intermittent and radiating, which aggravates on eating food and subsided after taking medication. She had undergone extraction of left second premolar tooth 8 weeks back and was referred for CT scan for further evaluation. On general examination the patient was anemic and weak. Extra-oral examination revealed a diffused tender swelling on left side of the mandible which was hard in consistency and overlying skin color was normal. On intra oral examination revealed erythematous mucosa with a draining intraoral sinus in the region of left second premolar tooth. Plain radiograph revealed soft tissue swelling with cortical erosions in the mandible.

Computed Tomography (CT) (Figure 1, 2 and 3) scan revealed cortical destruction with lamellated type of periosteal reaction in the mandible. There was cortical destruction of both lingual and buccal cortical plates. Pattern of destruction was diffuse lytic and mixed type. Soft tissue thickening was also seen in the left mandibular region (Figure 2). Clinical and radiological diagnosis of chronic suppurative osteomyelitis of mandible was made followed by surgical debridement. Histopathology confirmed the diagnosis of chronic suppurative osteomyelitis. Culture sensitivity was negative for mycobacterium tuberculosis. Patient was kept on antibiotics, analgesics and betadine mouth wash. On follow-up, the patient responded to the treatment. 


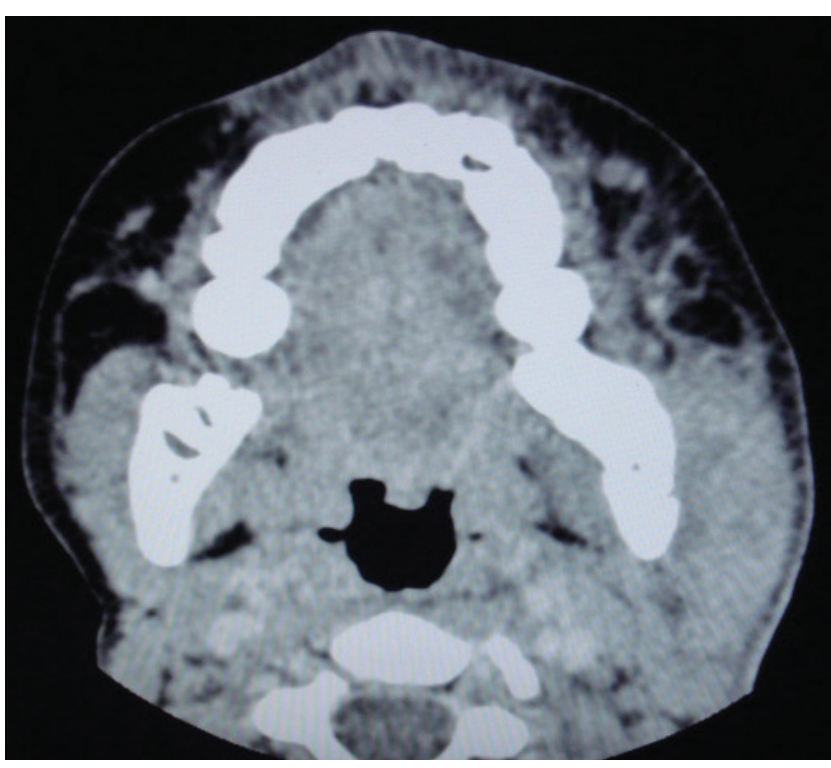

Figure 1: Axial section CT image (soft tissue window) showing soft tissue swelling in left mandibular region.
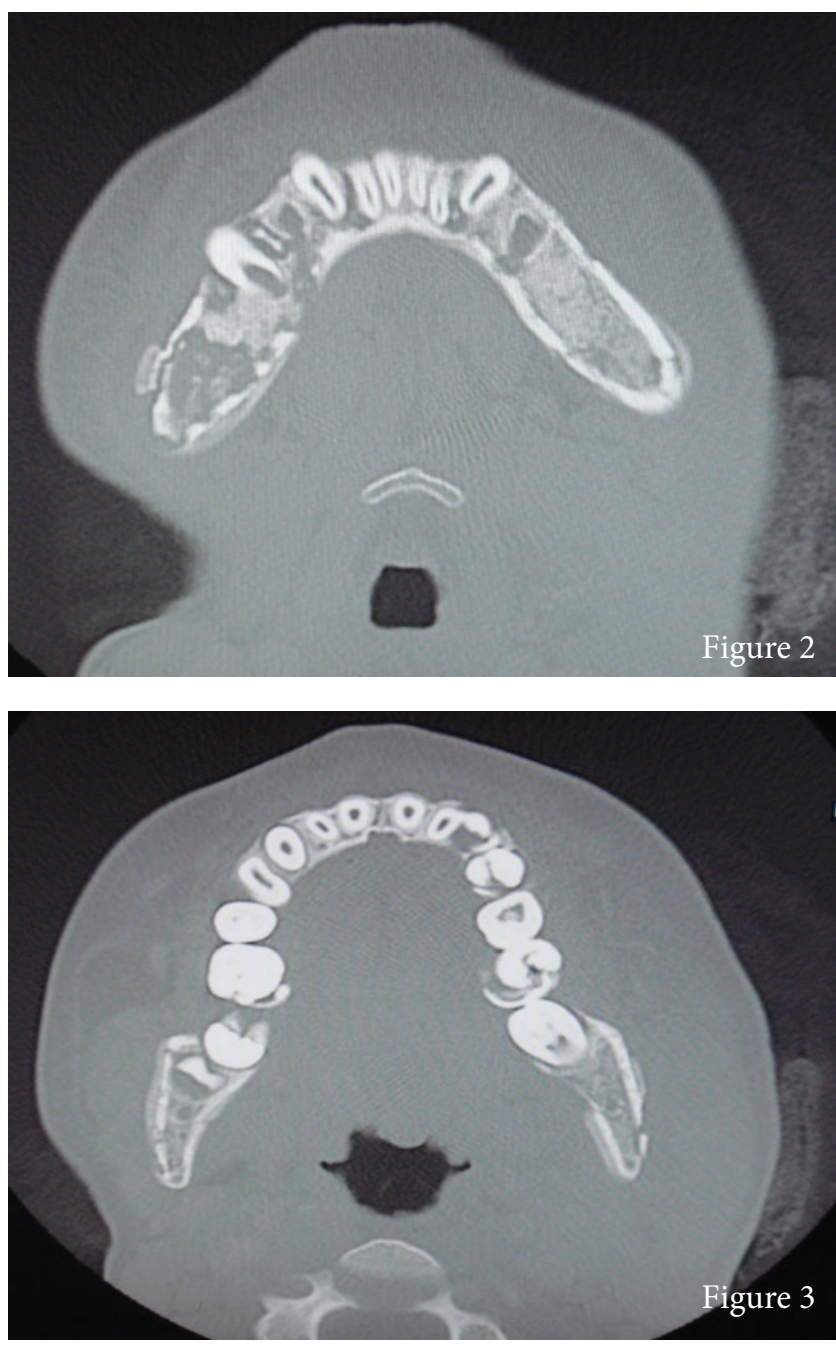

Figure 2 and 3: Axial section CT image (bone window) showing cortical destruction with lamellated type of periosteal reaction in the mandible

\section{Discussion:}

Mandibular osteomyelitis is a rare condition, which has been associated with multiple systemic diseases including diabetes, autoimmune diseases, malignancies, malnutrition and acquired immunodeficiency syndrome. Several causes have been demonstrated, such as dento-alveolar infection, trauma, operative procedures, radiation, neoplasia and genetic conditions. ${ }^{6}$ Steroids, chemotherapeutic agents and bisphosphonates are the other causes of mandibular osteomyelitis. ${ }^{7}$ Due to poor blood supply of mandible as compared to the maxilla and also due to the dense mandibular cortical bone, mandible is more susceptible to damage and infection at the time of extraction of tooth, that's why osteomyelitis of the mandible is more frequently encountered. ${ }^{8,9}$ Osteomyelitis has a range of clinical manifestations depending on the virulence of the infecting organisms, host resistance and the reaction of the periosteum to inflammation. ${ }^{10}$

Classically, patient with osteomyelitis of the mandible would experience pain and swelling over the affected side of the face. ${ }^{11}$ Other features may include discharge / drainage, trismus and paraesthesia in the distribution of the inferior alveolar nerve. ${ }^{12}$

CT is usually indicated when there is extension of the infection into the adjacent soft tissue and fascial spaces which could be the presenting clinical symptom. Transcortical extension of the inflammatory process can result in cortical destruction, fistulization and periosteal reaction; all these changes can be evaluated very well by $\mathrm{CT} .{ }^{13}$ Mandibular osteomyelitis is classified into four basic patterns: lytic, sclerotic, mixed and sequestrum pattern. ${ }^{14}$ Spiculated mixed pattern osteomyelitis with cortical plate abnormalities may initiate masticator spaces inflammation.

Treatment of osteomyelitis of the jaws includes elimination of the cause, incision and drainage, sequestrectomy, saucerization, decortication, resection of the jaw, antibiotics and hyperbaric oxygen. ${ }^{15}$ Antibiotic therapy should be instituted at the earliest moment.

\section{Conclusion:}

Although of rare occurrence, the differential diagnosis of manidbular osteomyelitis must always be kept in mind by clinicians, when a patient presents with trismus and soft tissue swelling in the region of mandible. CT is usually indicated when there is extension of the infection into the adjacent soft tissue and fascial spaces which could be the presenting clinical symptom.

\section{Conflict of interest: None}




\section{References:}

1. Arunkumar JS, Naik AS, Prasad KC, et al. Role of nasal endoscopy in chronic osteomyelitis of maxilla and zygoma: a case report. Case Report Med 2011: Article ID 802964.

2. Kushner GM, Alpert B. Osteomyelitis and osteoradionecrosis.In: Miloro M, Ghali GE, Larsen PE, Waite PD, eds. Peterson's Principles of Oral and Maxillofacial Surgery, 2nd ed. London:BC Decker; 2003:313-21.

3. Fonseca RJ, Turvey TA, Betts NJ. Oral and Maxillofacial Surgery, 1st ed. Philadelphia: WB Saunders; 2000:485-90.

4. Yavuz MS, Kaya GS, Yalçin E, et al. Mandibular bone necrosis caused by use of arsenic paste during endodontic treatment: two case reports. Int Endod J 2008;41:633-7.

5. Ozmeriç N. Localized alveolar bone necrosis following the use of an arsenical paste: a case report. Int Endod J 2002;35:295-99.

6. KoorbuschGF, Fotos P, Goll KT. Retrospective assessment of osteomyelitis. Etiology, demographics, risk factors, and management in 35 cases. Oral Surg Oral Med Oral Pathol 1992;74:149-54.

7. Kushner G M, Alpert B. Osteomyelitis and osteoradionecrosis. In: Miloro M, Ghali GE, Larsen PE, Waite PD, eds. Peterson's Principles of Oral and Maxillofacial Surgery, 2nd ed. London: BC Decker; 2003:31321.
8. Barry CP, Ryan CD, Stassen LF. Osteomyelitis of the maxilla secondary to osteopetrosis: a report of 2 cases in sisters. J Oral Maxillofac Surg 2007;65:144-7.

9. Baltensperger M, Eyrich G. Osteomyelitis of the Jaws: Springer Berlin, Heidelberg. November 07, 2008. 5.

10. Jones J, Amess TR, Robinson PD. Treatment of chronic sclerosing osteomyelitis of the mandible with calcitonin: a report of two cases. Br J Oral Maxillofac Surg 2005;43:173-6.

11. Mohammed-Ali RI, Collyer J, Garg M. Osteomyelitis of the mandible secondary to pericoronitis of an impacted third molar. Dent Update 2010;37:106-8.

12. Uche C, Mogyoros R, Chang A, et al. Osteomyelitis of the jaw: a retrospective analysis. Internet $\mathrm{J}$ Infect Dis $2009 ; 7(2)$.

13. Yeoh SC, MacMahon S, Schifter M. Chronic suppurative osteomyelitis of the mandible: Case report. Aust Dent J 2005;50:200-3.

14. Taori KB, Solanke R, Mahajan SM, et al. CT evaluation of mandibular osteomyelitis. Indian J Radiol Imaging 2005; 15:447-51.

15. Kushner G M, Alpert B. Osteomyelitis and osteoradionecrosis. In: Miloro M, Ghali GE, Larsen PE, Waite PD, eds. Peterson's Principles of Oral and Maxillofacial Surgery, 2nd ed. London: BC Decker; 2003:31321. 